\title{
Review: both Gram stain and urine dipstick analysis were accurate in diagnosing urinary tract infection in children
}

Gorelick MH, Shaw KN. Screening tests for urinary tract infection in children: a meta-analysis. Pediatrics 1999 Nov 5;104:e54. QUESTION: How accurate are rapid diagnostic tests for diagnosing urinary tract
infection (UTI) in children?

\section{Data sources}

Studies were identified by searching Medline (1966-98) using the terms urine, urinalysis, pyuria, reagent strips, bacteriuria, urinary tract infections, pyelonephritis, and terms related to a paediatric population. Reference lists of relevant studies were scanned, and experts were contacted.

\section{Study selection}

Published English language studies were selected if they reported original data; $\geqslant 1$ of urine dipstick (leukocyte esterase, nitrite, or both), Gram stain of unspun urine, or enhanced urinalysis tests were used in the diagnosis of UTI; children $\leqslant 12$ years of age were included or evaluated separately; urine culture was the diagnostic standard; the diagnostic standard and screening tests were done on all patients and the results were compared; and the definition for a positive screening test result was given.

\section{Data extraction}

Data were extracted on participant age, colony count used to define UTI, whether a general or special population was used, methodologic concerns, tests performed, sensitivity and specificity for diagnosing UTI, rate of UTI, and method of obtaining specimens.

\section{Main results}

1489 studies were identified and 26 met the selection criteria. Weighted sensitivities, specificities, and likelihood ratios for tests are shown in the table. For predicting a positive urine culture, the presence of any bacteria on a Gram stained urine specimen had the best combination of sensitivity and specificity, with the highest sensitivity. The dipstick test performed nearly as well, with a slightly lower sensitivity for the presence of any nitrite or leukocyte esterase and a slightly higher specificity for the presence of both nitrite and leukocyte esterase.

\section{Conclusion}

The Gram stain test of unspun urine and the urine dipstick test performed similarly in detecting urinary tract infections in children.

\section{COMMENTARY}

Diagnostic guidelines for UTI in children are confusing because primary care textbooks often rely exclusively on culture of catheterised specimens as criteria for a positive diagnosis of UTI. ${ }^{2}$ Although this is supported by microbiologic evidence, it is of little help in children who are uncomfortable or febrile, have abnormal urine tests, or whose culture results will take 48 hours to obtain.

This review by Gorelick and Shaw included a large number of published studies with clear and credible criteria for inclusion. Data were then examined by age and culture colony count in an attempt to explain the variability of results, which neither fully explained. The findings of this review are supported by a similar meta-analysis published 6 months earlier by the American Academy of Pediatrics on children $\leqslant 2$ years of age. ${ }^{3}$. They reported a sensitivity and specificity of a positive leukocyte esterase test or a positive nitrite test of $92 \%$ and $70 \%$, respectively, compared with $100 \%$ and $70 \%$, respectively, for culture of a bagged specimen. The American Academy of Pediatrics study found poor reliability with microscopy of spun urine and suggested that this was because of variation in the expertise of the people who performed the microscopy.

The results of this study are relevant to paediatric and family nurse practitioners and nurses in primary care settings. Nurse practitioners make treatment decisions and nurses in primary care explain treatment plans to patients and families. In addition to the accuracy of the diagnostic technique, primary care practitioners should also consider patient presentation, comfort of the patient and parents, and the need for invasive procedures when making decisions. Stephanie Wright, RN, PhD, CFNP Associate Professor Georgetown University Washington, DC, USA

1 Genitourinary disorders. In: C E Burns, N Barber, M A Brady, et al [eds]. Pediatric primary care: a handbook for nurse practitioners. Philadelphia, Pennsylvania: WB Saunders, 1996:695-716.

2 Urinary tract infections. In: R A Hoekelman [ed]. Primary pediatric care. St Louis, Missouri: Mosby, 1997:1640-4.

3 Downs SM. Technical report: urinary tract infections in febrile infants and young children. The Urinary Tract Subcommittee of the American Academy of Pediatrics Committee on Quality Improvement. Pediatrics 1999;103:e54.
Source of funding: Maternal and Child Health Bureau, US Department of Health and Human Services.

For correspondence: Dr $M$ H Gorelick,

Division of Emergency Medicine, A I duPont Hospital for Children, 1600 Rockland Road, Wilmington, DE 19899 USA. Fax +1302651 4227.
Test characteristics for the diagnosis of urinary tract infection in children

\begin{tabular}{|c|c|c|c|c|c|}
\hline Test & $\begin{array}{l}\text { Number of } \\
\text { studies }\end{array}$ & $\begin{array}{l}\text { Weighted } \\
\text { sensitivity }\end{array}$ & $\begin{array}{l}\text { Weighted } \\
\text { specifi city }\end{array}$ & $\begin{array}{l}\text { Weighted } \\
+ \text { LR }^{*}\end{array}$ & $\begin{array}{l}\text { Weighted } \\
- \text { LR }^{*}\end{array}$ \\
\hline Dipstick, any nitrite only & 13 & $50 \%$ & $98 \%$ & 25 & 0.51 \\
\hline Dipstick, any $\mathrm{LE}^{\dagger}$ only & 7 & $83 \%$ & $84 \%$ & 5 & 0.2 \\
\hline Dipstick, any nitrite or $\mathrm{LE}^{\dagger}$ & 9 & $88 \%$ & $93 \%$ & 13 & 0.13 \\
\hline Dipstick, both nitrite and $\mathrm{LE}^{\dagger}$ & 5 & $72 \%$ & $96 \%$ & 18 & 0.29 \\
\hline Gram stain, any organisms & 5 & $93 \%$ & $95 \%$ & 19 & 0.07 \\
\hline Enhanced analysis of spun urine & 5 & $67 \%$ & $79 \%$ & 3 & 0.42 \\
\hline Enhanced analysis of unspun urine & 9 & $77 \%$ & $89 \%$ & 7 & 0.26 \\
\hline
\end{tabular}

*Likelihood ratios (LRs) defined in Glossary and calculated from data in article. ${ }^{\dagger} \mathrm{LE}=$ leukocyte esterase. 